\title{
Low-temperature electrical-transport properties of single-crystal bismuth films under pressure
}

\author{
Mei Lu and R. J. Zieve \\ The James Franck Institute and Department of Physics, The University of Chicago, Chicago, Illinois 60637 \\ A. van Hulst \\ Delft Institute of Microelectronics and Submicron Technology, Delft University of Technology, \\ Lorentzweg 1, 2628 CJ Delft, The Netherlands \\ H. M. Jaeger and T. F. Rosenbaum \\ The James Franck Institute and Department of Physics, The University of Chicago, Chicago, Illinois 60637 \\ S. Radelaar \\ Delft Institute of Microelectronics and Submicron Technology, Delft University of Technology, \\ Lorentzweg 1, 2628 CJ Delft, The Netherlands
}

(Received 23 February 1995; revised manuscript received 10 May 1995)

\begin{abstract}
We report an investigation of the low-temperature electrical transport properties of bismuth films under applied hydrostatic pressure. Films with their trigonal axis perpendicular to the film plane and thicknesses of 30,50 , and $500 \mathrm{~nm}$ were grown by molecular-beam epitaxy on $\mathrm{BaF}_{2}$ substrates. At $500 \mathrm{~nm}$ thickness the behavior resembles that of bulk Bi. From the observed Shubnikov-de Haas oscillations we find a pressureinduced decrease in extremal Fermi cross section. For the 30-nm film, we obtain the low-temperature carrier densities for electrons and holes together with the corresponding mobilities from magnetoconductance data at pressures up to $20 \mathrm{kbar}$. We find that pressure strongly reduces the surface-induced excess hole concentration, clearly revealing a finite electron concentration at high pressures. We discuss our results within the context of a possible semimetal-semiconductor transition in thin Bi films.
\end{abstract}

\section{INTRODUCTION}

When the typical dimensions of an electronic conductor are reduced to a scale comparable to the Fermi wavelength of the charge carriers, the resulting carrier confinement leads to a quantum behavior not present in the corresponding bulk system. ${ }^{1}$ Changes in the electronic transport behavior due to quantum confinement were observed experimentally in the 1960s, in thin semimetallic bismuth films. ${ }^{2}$ In $\mathrm{Bi}$ the low carrier concentration gives rise to a Fermi wavelength close to $40 \mathrm{~nm}$, significantly larger than in metals. Together with a small effective mass and a long mean-free path, this has made $\mathrm{Bi}$ a material of choice in the search for manifestations of quantum transport behavior. ${ }^{3-6}$ One particular consequence of quantum confinement in $\mathrm{Bi}$ concerns the existence of a semimetal-semiconductor (SMSC) transition. This transition was postulated by Sandomirskii ${ }^{1}$ and Lutskii and Kulik, ${ }^{7}$ and has remained a controversial issue despite many experimental $^{3-5,7-10}$ and theoretical ${ }^{8,11-13}$ efforts. Most recently, Hoffman et al. ${ }^{14}$ claim to have identified experimentally the SMSC transition in epitaxial thin Bi films.

In order to test for the SMSC transition, work to date has concentrated on varying the film thickness. In $\mathrm{Bi}$, the transition should occur when the energy shift due to confinement becomes large enough to raise the lowest electron subband to an energy higher than that of the uppermost hole subband. ${ }^{8,10-13}$ Since this is expected to occur in the thickness range $20-30 \mathrm{~nm}$, a major obstacle has been fabricating thin films of sufficiently high quality. In particular, island growth or grain boundaries could mask the appearance of quantum confinement. ${ }^{15}$ Only recently have molecular-beam epitaxy (MBE) techniques been developed for the growth of Bi films. ${ }^{16,17}$

As the film thickness is reduced, a new problem arises, first observed by Komnik and co-workers. ${ }^{4,5}$ Due to band bending and the increasing importance of surface states, the effective carrier density rises significantly as the film thickness decreases. Komnik and co-workers ${ }^{4,5}$ fit the total carrier density in their films to $n=n_{0}+n_{2} / d$, where $d$ is the film thickness and $n_{0}$ and $n_{2}$ are the bulk and surface carrier densities, respectively. They found $n_{0}=2.5 \times 10^{17} \mathrm{~cm}^{-3}$, close to the bulk carrier density in $\mathrm{Bi}$ from other experiments, ${ }^{18,19}$ and $n_{2}=2.75 \times 10^{12} \mathrm{~cm}^{-2}$. For $d$ near 30 $\mathrm{nm}$ these extra carriers dominate the transport properties, and any band gap which may open in the density of states away from the surface is impossible to observe through conductivity measurements.

Clearly the surface states are an obstacle to measuring the SMSC transition as a function of thickness alone. Here we describe a different approach, using hydrostatic pressure to drive Bi films near the SMSC transition. In bulk Bi pressure is known ${ }^{20}$ to reduce the band overlap. Extrapolation of the available data indicates a vanishing of the carrier density and, thus, the possibility of observing a SMSC transition around 20-25 kbar. Combining externally applied pressure with size confinement, we prepare single-crystalline Bi films with thicknesses just above the predicted zero-pressure critical values and use pressure to further tune the energy bands. To the best of our knowledge, this is the first investigation of the effect of pressure on the electrical transport properties of thin Bi films. 
We find that hydrostatic pressure can compensate for the thin-film carrier density increase. We extract the pressure dependence of the carrier concentration from fits to magnetoconductance data. Low-field magnetoconductance measurements are sensitive primarily to the high mobility carriers, while low mobility carriers give rise to a nonvanishing, residual conductance in the high-field limit. Shubnikov-de Haas oscillations supply additional information on the Fermi surface and verify the quality of our films.

The paper is organized as follows. In Sec. II we describe the sample fabrication and pressure cell characteristics. Our magnetoconductance measurements are presented in Secs. III-V. In the concluding Sec. VI, we discuss our results within the context of a possible semimetal-semiconductor transition.

\section{EXPERIMENTAL PROCEDURE}

Thin single-crystalline Bi films, with trigonal axis perpendicular to the film plane, were grown by $\mathrm{MBE}$ on $\mathrm{BaF}_{2}$ single-crystal substrates cleaved along the (111) plane. The cleaving produced substrate surfaces that were atomically flat on a microscopic scale, but had approximately parallel steps of several $\mu \mathrm{m}$ height spaced a few mm apart. ${ }^{21}$ Prior to growth, the substrates were heated to $500{ }^{\circ} \mathrm{C}$ and $450{ }^{\circ} \mathrm{C}$ for several minutes in the introduction and the growth chamber, respectively, of the $\mathrm{MBE}$ system and then cooled to $110^{\circ} \mathrm{C}$. At this temperature, Bi films were grown at a rate of $450 \mathrm{~nm} / \mathrm{h}$, as calibrated by weight measurements of the samples before and after growth. Reflection high-energy electron diffraction (RHEED) was used to examine the surface of the deposited layers. The RHEED patterns exhibited two-dimensional (2D) character (lines), with only a small additional 3D component (spots), indicating a very smooth surface. Both RHEED and x-ray diffraction indicate that the films are single crystalline. Details of the growth procedure, as well as an analysis of the resulting Bi films with scanning electron microscopy and atomic force microscopy, are described elsewhere. ${ }^{17,22}$

We describe transport measurements on films with thicknesses of 30, 50, and $500 \mathrm{~nm}$. The 30-nm- and 50-nm-thick samples were capped in situ with a 50-nm-thick amorphous $\mathrm{Si}$ overlayer in order to prevent oxidation. These thinner samples are close to the critical thickness for the SMSC transition. The 500-nm-thick sample provides a comparison with the available data on the pressure dependence of transport in bulk crystals.

Pressure was applied hydrostatically by a piston-clamp cell made of $\mathrm{BeCu}$ and $\mathrm{WC}{ }^{23}$ The cylindrical pressure chamber had an inner diameter of $\frac{1}{4}$ in and a height of $\frac{3}{4}$ in and contained a squeezable Teflon bucket that enclosed both the sample and a $1 \times 1 \times 1.5-\mathrm{mm}^{3}$ high-purity $\mathrm{Pb}$ manometer. ${ }^{24}$ Copper wires were attached to the sample and the manometer for resistance measurements, and were fed out of the pressure chamber through a small, epoxy-sealed access hole. Silicone oil was used as the pressure medium. For all magnetotransport measurements on the sample, we employed standard four-terminal or van der Pauw ${ }^{25}$ current and voltage lead configurations and a Linear Research LR-400 ac resistance bridge. The pressure cell was mounted inside a toploading ${ }^{3} \mathrm{He}$ cryostat equipped with an 8 -T superconducting

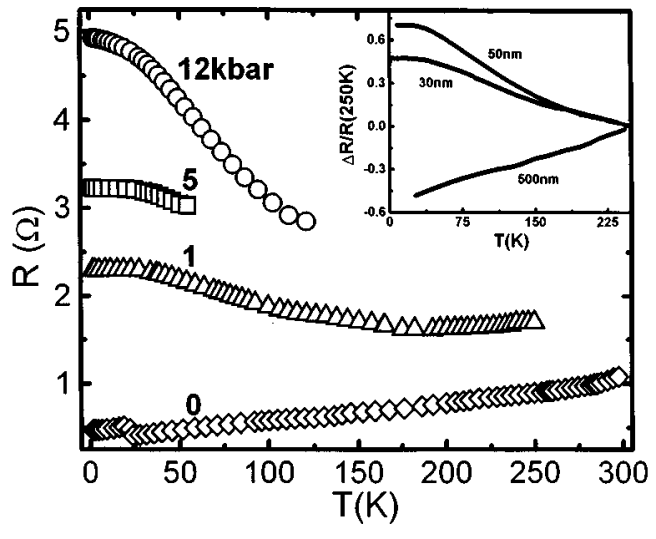

FIG. 1. Temperature dependence of the electrical resistance, $R(T)$, for the 500-nm-thick Bi film at pressures of $0 \mathrm{kbar}$ (diamonds), 1 kbar (triangles), $5 \mathrm{kbar}$ (squares), and $12 \mathrm{kbar}$ (circles). The inset compares the zero-pressure normalized resistance $\Delta R / R(250 \mathrm{~K})$, where $\Delta R=R(T)-R(250)$, for the 500-, 50-, and 30-nm-thick films.

magnet. All pressure changes were made at room temperature. After each new setting the cell was top loaded into the cryostat and cooled again.

\section{TEMPERATURE DEPENDENCE OF RESISTANCE}

Figure 1 shows the temperature dependence of the electrical resistance, $R(T)$, of the 500-nm-thick Bi film at several pressures. At low applied pressure, the resistance decreases with decreasing temperature and the sample exhibits metallic behavior with a residual resistance ratio, $R(300 \mathrm{~K}) / R(4.2 \mathrm{~K})$, close to 2 . The small jump near $25 \mathrm{~K}$ in the zero-pressure data was observed only during the first cooldown. $R(T)$ traces for subsequent cooldowns were always reproducible and smooth. The jump in $R(T)$ is most likely the result of film rupture along terrace edges on the substrate, where the film had to bridge an abrupt height difference of several $\mu \mathrm{m} .^{21}$ In order to minimize these jumps, samples with large terraces were selected and all electrical connections were made on the same terrace.

As pressure was applied, the temperature coefficient of resistance (TCR), $d R / d T$, changed from positive to negative. At low temperatures, $R(T)$ flattened off towards a residual resistance, indicative of a metallic ground state. With increasing pressure, the residual resistance increased rapidly and the temperature onset of the plateau decreased.

The form of the resistance traces, $R(T)$, is complicated for several reasons. In bulk $\mathrm{Bi}$ three identical, anisotropic electron pockets and one hole pocket contribute to the overall conductivity. In thin films, an additional factor is the possibility of significant parallel conduction through carriers residing along the interfaces. Finally, the carrier concentration and the mobility, both of which affect the resistance, have opposite temperature dependences: as the temperature is lowered, the carrier concentration decreases while the mobility increases. These opposing influences can produce minima or maxima in $R(T)$. The $P=1$ kbar curve of Fig. 1, for example, has a minimum near $180 \mathrm{~K}$. Even in high purity, bulk Bi crystal pressure changes the magnitude and temperature dependence of both the carrier concentration and the 


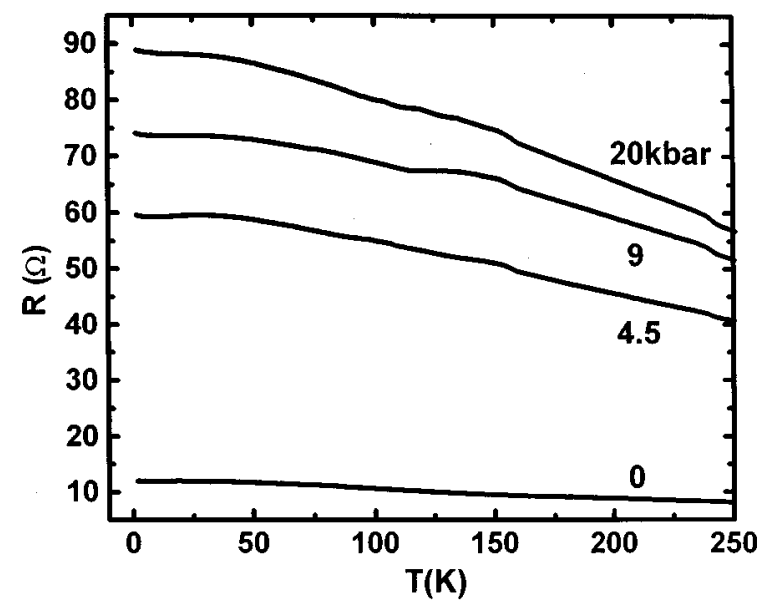

FIG. 2. Temperature dependence of the resistance for the 30nm-thick Bi film up to 20 kbar.

mobility, leading to a sign change in the TCR near $13 \mathrm{kbar}^{26}$ Our data show the TCR sign change at a lower pressure, approximately 2 kbar. A likely explanation is that sample imperfections in the film reduce the mobility and decrease its temperature dependence, which increases the influence of the carrier concentration.

The inset to Fig. 1 shows the zero-pressure temperature dependence of the 30-, 50-, and 500-nm films. Both the nonmonotonic character of these curves with film thickness and the negative TCR of the thinner films agree with previous measurements on $\mathrm{Bi}$ films. ${ }^{4,9}$ Like the pressure measurements described above, these results can be attributed to the competing effects on resistance of carrier concentration and mobility. ${ }^{4}$ The negative TCR does not imply that a band gap has opened up, either in the thin films or under pressure. Indeed, the observed flattening off of $R(T)$ at the lowest temperatures and its weak, nonexponential decrease at higher temperatures (see also Fig. 2) are incompatible with activated transport in the presence of a gap.

The $R(T)$ curves give an indication of sample quality. In earlier work, individual films on the order of $500 \mathrm{~nm}$ thick did not have strictly positive $d R / d T$, but rather a minimum in $R(T)$ near $150 \mathrm{~K}^{4}$ The temperature of the minimum was found to decrease as the film quality improved. This suggests that our 500-nm film, in which the minimum has been driven to zero temperature, is of significantly higher quality than those of Ref. 4.

In Fig. 2 we show $R(T)$ under pressure for the $30-\mathrm{nm}$ sample. The residual resistance increases with pressure, as it does for the 500-nm film. However, the TCR, which changes steadily with pressure in the thicker film, is essentially constant here. Once again, this supports the idea that pressure and small size both reduce the temperature dependence of the mobility. For this extremely thin film, the mobility is nearly temperature independent even at zero pressure, so pressure has little effect on the TCR.

\section{SHUBNIKOV-de HAAS OSCILLATIONS}

The carrier types, densities, and mobilities as a function of thickness and pressure can be obtained from an analysis of the magnetic-field dependence of the electrical transport.
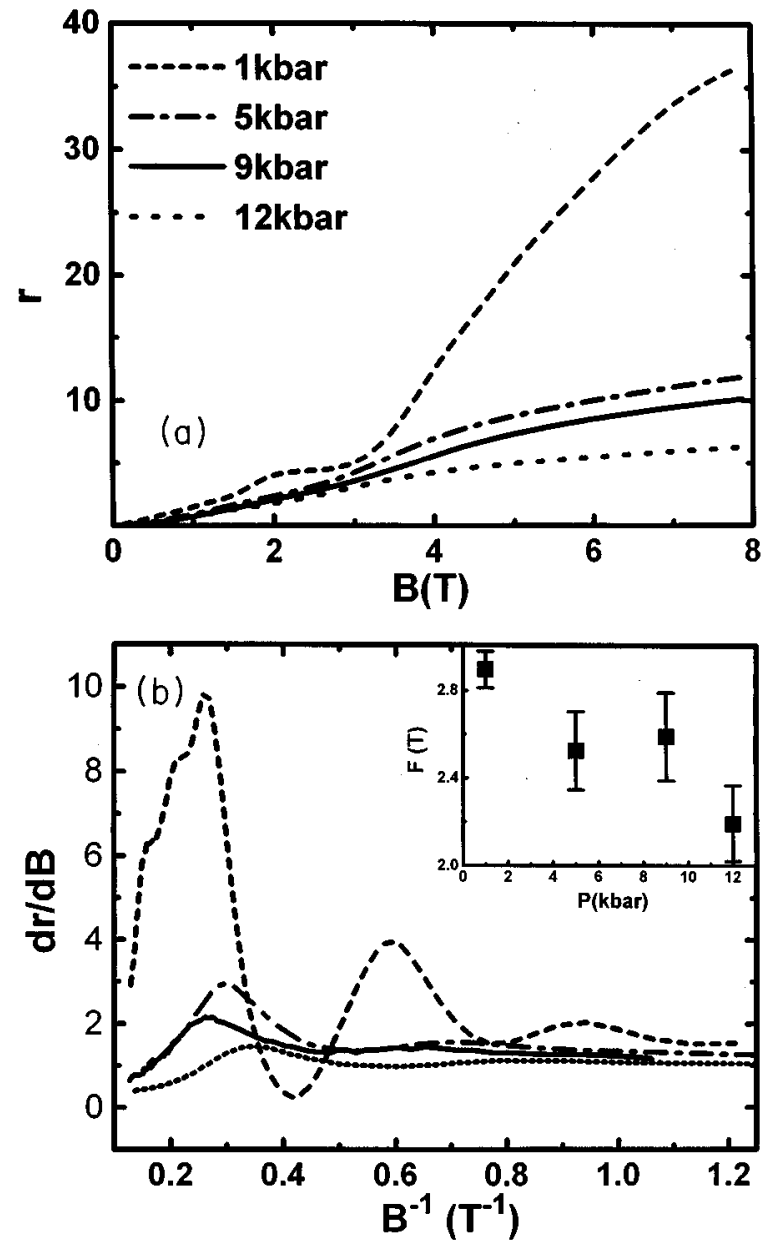

FIG. 3. (a) Normalized magnetoresistance, $r=[R(B)$ $-R(B=0)] / R(B=0)$, as a function of applied magnetic field, $B$. $B$ is parallel to the film plane and $T=1.7 \mathrm{~K}$. (b) Derivative of the normalized magnetoresistance, $d r / d B$, versus $1 / B$, for the data in (a) (the line types correspond to the same hydrostatic pressures). Shubnikov-de Haas oscillations are clearly visible. The normalized magnetoresistance for the 1-kbar (dashed) trace has been multiplied by 0.6 for this plot. The inset shows the decrease of the oscillation period, $F$, with increasing hydrostatic pressure, $P$.

One indication of carrier density comes from Shubnikov-de Haas $(\mathrm{SdH})$ oscillations of the magnetoresistance, $R(B, T)$. $\mathrm{SdH}$ oscillations show a characteristic period, $F$, when plotted against inverse magnetic field, $1 / B . F$ is proportional to the external cross section through the Ferm surface perpendicular to the direction of the applied magnetic field. The size of the Fermi surface in turn is related to the density of carriers in the relevant hole or electron pockets. In the following, the subscripts $\perp$ or $\|$ denote $B$ perpendicular or parallel to the film plane, respectively.

For both field directions, the 500-nm-thick sample shows $\mathrm{SdH}$ oscillations superimposed on a large background magnetoresistance. We plot in Fig. 3(a) the normalized magnetoresistance $r=[R(B, T)-R(0, T)] / R(0, T)$ in parallel field at $T=1.7 \mathrm{~K}$. The data were averaged over both current and field directions. To show the oscillations more clearly, we plot in Fig. 3(b) the derivative of the magnetoresistance, $d r / d B$, against inverse magnetic field. These oscillations are the cleanest reported to date in any $\mathrm{Bi}$ film with submicrome- 
ter thickness, ${ }^{10,16}$ a further indication of high sample quality. The oscillation period $F_{\|}=2.8 \mathrm{~T}$ at low pressure. In bulk Bi, $F_{\|}=1.2 \mathrm{~T}$ for electrons in the smaller orbit perpendicular to the bisectrix axis, and $F_{\|}=20 \mathrm{~T}$ for holes. We assume that our measured value corresponds to electron pockets with extremal cross sections enhanced compared to bulk Bi. Much of this enhancement is likely due to an increase in band overlap near the film interfaces; as we explain below, we find an increase in the period $F_{\perp}$ for perpendicular field as well. A smaller factor is that the bulk period, measured on wellaligned single-crystal samples, corresponds to the smallest possible orbit detected with a field in the sample plane. We did not align the in-plane crystal axes on assembling the pressure cell. In-plane misorientation can enlarge $F_{\|}$by up to $15 \%$. Out-of-plane misalignment is about $10 \%$ or less and contributes only an additional $2 \%$ enlargement.

At first glance strain, induced either by pressure or by the growth process, is a plausible source of band bending and the resulting increase in band overlap. However, measurements of the $\mathrm{SdH}$ frequency before applying any pressure show similar enhancement over the bulk value, so pressureinduced strain is not causing the enhancement. Furthermore, comparable thickness films have similar $\mathrm{SdH}$ frequencies when grown on either $\mathrm{Si}$ or $\mathrm{Bi}_{1-x} \mathrm{Sb}_{x}$ layers. ${ }^{17,22}$ Since these materials have different lattice constants, the enhancement source is not simply dependent on the growth procedure.

For comparison to bulk carrier concentrations, we use $F_{\perp}$ rather than $F_{\|} . F_{\perp}$ increases from $6.4 \mathrm{~T}$ in bulk Bi to $9 \mathrm{~T}$ in our 500-nm sample, a factor of 1.4. With the assumption that the carrier ellipsoids change proportionately the same amount in all directions, the corresponding carrier density is $n \approx n_{0}\left(1.4^{3 / 2}\right)=4.2 \times 10^{17} \mathrm{~cm}^{-3}$. (We use $n_{0}=2.75 \times 10^{17}$ $\mathrm{cm}^{-3}$ from Ref. 18.) However, since the bulk Fermi surface cross sections for electrons and holes are almost identical in perpendicular field, identification of the carrier type responsible for the oscillations is impossible.

We measure the pressure dependence of $F_{\|}$rather than $F_{\perp}$ because the $\mathrm{SdH}$ oscillations have larger amplitude in parallel field. As shown in the inset to Fig. 3(b), hydrostatic pressure of 12 kbar decreases the cross section of the Fermi surface by about $20 \%$ in the $500-\mathrm{nm}$ film. The cross section in bulk Bi also decreases under pressure, by about $40 \%$ for the same $12 \mathrm{kbar}^{27}$ In addition to the change in $F_{\|}$, the amplitude of the SdH oscillations decreases sharply with increasing pressure. The amplitude depends exponentially on the Fermi energy, the cyclotron frequency, and the singleparticle relaxation rate, ${ }^{28}$ any of which may change with pressure. From our measurements of $F_{\|}$we know that the product $E_{F} m^{*}$ increases, where $E_{F}$ is the Fermi energy and $m^{*}$ the effective mass. This can account for the pressure dependence of the amplitude shown in Fig. 3(b), except for the sharp change between 1 and $5 \mathrm{kbar}$. A small deformation during the early stages of sample compression, which could increase the scattering rate, is probably responsible for the initial large decrease in amplitude.

We find that the $\mathrm{SdH}$ oscillation amplitude is strongly reduced in the thin-film samples, so that a reliable value for the oscillation period can be obtained only for the 50-nm film and in the absence of applied pressure. We find $F_{\perp}=19.6 \mathrm{~T}$ at $50 \mathrm{~nm}$, an enhancement by a factor of 3 over bulk, as opposed to 1.4 for the $500-\mathrm{nm}$ film. As expected, the

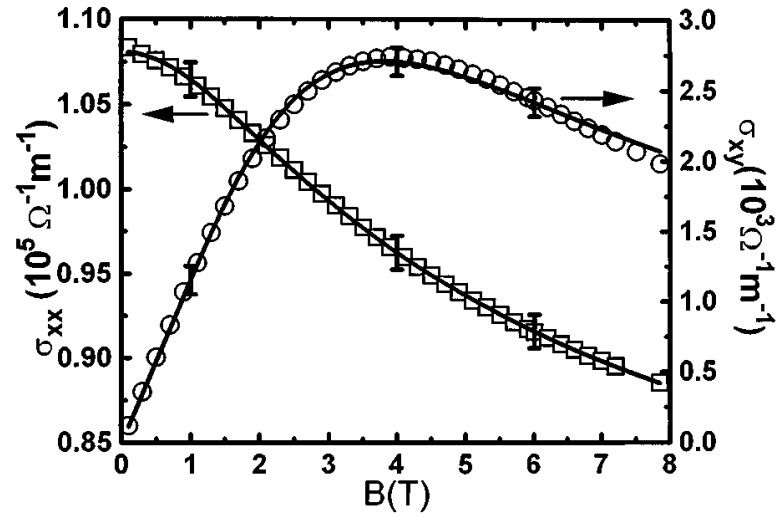

FIG. 4. Magnetoconductivity ( $\sigma_{x x}$, squares) and Hall conductivity ( $\sigma_{x y}$, circles) for the 30-nm-thick Bi film at $P=20 \mathrm{kbar}$ and $T=1.5 \mathrm{~K}$ with magnetic field perpendicular to the film plane. The lines through the data correspond to simultaneous, five-parameter fits of $\sigma_{x x}$ and $\sigma_{x y}$ to Eqs. (1). The resulting parameter values are given in Table I. The error bars are representative of the variation of the fitting parameters within the error margins in Table I.

influence of the film surfaces on the band structure is greater for the thinner film. Assuming, as for the 500-nm film, that the carrier pockets grow proportionately in all directions, we estimate a carrier density $n$ or $p \approx n_{0}\left(3^{3 / 2}\right)=14 \times 10^{17}$ $\mathrm{cm}^{-3}$.

\section{MAGNETOCONDUCTIVITY AND HALL MEASUREMENTS}

Although the SdH oscillations are difficult to observe in the thinner films, the carrier densities can be extracted instead from magnetoconductivity data. This method also gives the carrier mobilities, and distinguishes between the carrier densities of holes and electrons. The magnetoconductivity, $\sigma_{x x}$, and the Hall conductivity, $\sigma_{x y}$, were measured with magnetic field perpendicular to the film plane. Figure 4 shows representative magnetoconductance data for the 30nm-thick film, taken at $20 \mathrm{kbar}$ and $1.5 \mathrm{~K}$. We note that $\sigma_{x x}$ does not approach zero in the high-field limit but rather extrapolates to a finite value. ${ }^{29}$

The conductivities were fit to the expressions

$$
\begin{gathered}
\sigma_{x x}=\frac{n e \mu_{n}}{1+\mu_{n}^{2} B^{2}} \frac{p e \mu_{p}}{1+\mu_{p}^{2} B^{2}}+c, \\
\sigma_{x y}=-\frac{n e \mu_{n}^{2} B}{1+\mu_{n}^{2} B^{2}}+\frac{p e \mu_{p}^{2} B}{1+\mu_{p}^{2} B^{2}} .
\end{gathered}
$$

Here $n$ and $p$ are the electron and hole concentrations, $\mu_{n}$ is the effective electron mobility, $\mu_{p}$ is the hole mobility, and $c$ is a constant. The first two terms in each equation are standard expressions derived from the conductivity tensor in the presence of a magnetic field. The constant third term in $\sigma_{x x}$ reflects the high-field, residual conductivity we observe in our data. The solid lines of Fig. 4 are least-squares fits. Equations (1) certainly do not provide the most general description, neglecting, e.g., the anisotropy in the electron mobilities found in bulk Bi. On the other hand, Eqs. (1) contain the 
TABLE I. Transport parameters for the 30-nm-thick film at hydrostatic pressures up to $20 \mathrm{kbar}$, obtained from fits to Eqs. (1). The data were taken at $1.5 \mathrm{~K}$. See the text for an explanation of the error margins.

\begin{tabular}{cccccc}
\hline \hline $\begin{array}{c}\text { Pressure } \\
(\mathrm{kbar})\end{array}$ & $\begin{array}{c}p \\
\left(10^{17} / \mathrm{cm}^{3}\right)\end{array}$ & $\begin{array}{c}\mu_{p} \\
\left(10^{4} \mathrm{~cm}^{2} / \mathrm{V} \mathrm{s}\right)\end{array}$ & $\begin{array}{c}n \\
\left(10^{17} / \mathrm{cm}^{3}\right)\end{array}$ & $\begin{array}{c}\mu_{n} \\
\left(10^{4} \mathrm{~cm}^{2} / \mathrm{V} \mathrm{s}\right)\end{array}$ & $\begin{array}{c}c \\
\left(10^{5} / \Omega \mathrm{m}\right)\end{array}$ \\
\hline 0 & $19 \pm 3$ & $0.33 \pm 0.05$ & $5 \pm 5$ & $0.4 \pm 0.4$ & $1.46 \pm 0.10$ \\
$2 \pm 1$ & $7.5 \pm 2.4$ & $0.35 \pm 0.05$ & $2.2 \pm 2.0$ & $0.37 \pm 0.15$ & $1.38 \pm 0.17$ \\
$4.5 \pm 0.5$ & $3.6 \pm 0.6$ & $0.38 \pm 0.03$ & $1.6 \pm 1.5$ & $0.39 \pm 0.10$ & $1.09 \pm 0.06$ \\
$9.0 \pm 0.5$ & $2.9 \pm 0.4$ & $0.27 \pm 0.03$ & $2.2 \pm 0.3$ & $0.25 \pm 0.04$ & $0.83 \pm 0.03$ \\
$20 \pm 2$ & $3.8 \pm 1.2$ & $0.24 \pm 0.04$ & $2.5 \pm 1.2$ & $0.23 \pm 0.03$ & $0.83 \pm 0.04$ \\
\hline \hline
\end{tabular}

least number of fitting parameters necessary to provide a consistently good fit to all our thin-film data. The parameter values obtained for the $30-\mathrm{nm}$ film are listed in Table I. We find that when the electronic anisotropy ${ }^{30}$ and a fielddependent surface carrier density ${ }^{19}$ are taken into account in more complex fitting formulas, the resulting values for the carrier densities and mobilities do not change significantly. The error margins cited in Table I include the range of values obtained from different fitting models. For the 9- and 20-kbar data the fits covered a field range up to $8 \mathrm{~T}$; at lower pressures, magnetic-field data extend only up to $1 \mathrm{~T}$.

The large background resistance underlying the $\mathrm{SdH}$ oscillations in the 500- and 50-nm films, as well as the residual, high-field magnetoconductivity [parametrized by the constant $c$ in Eq. (1)], point to a significant parallel conduction channel caused by additional, lower mobility carriers. This situation is known, for example, from GaAs semiconducting structures where it is attributed to interface states. ${ }^{31-33}$ In our films, the substrate/film interface is a likely source. Prior to sample growth, during the outgassing of the substrate, we detected a small fluorine signal in the mass spectrometer. If some fluorine left the $\mathrm{BaF}_{2}$ substrate surface, a small amount of $\mathrm{Ba}$ might have remained, which could account for extra conduction in parallel with the Bi film. For the $d=30 \mathrm{~nm}$ and $50 \mathrm{~nm}$ films, we find very similar values for the product $c d$ at zero applied pressure, namely, 0.44 and $0.48 \Omega^{-1}$, respectively. In $\mathrm{Bi} / \mathrm{Bi}_{1-x} \mathrm{Sb}_{x}$ heterostructures grown on the same substrate material $c d$ also stayed approximately constant for films of different thickness. ${ }^{22}$ These observations support the picture of a parallel conduction channel through surface states along the substrate/film interface.

As seen in Table I at zero pressure the carrier concentration of holes is much higher than that of electrons. The 50-nm film also has excess holes, with the electron concentration close to that in bulk $\mathrm{Bi}$ and the hole concentration consistent with the enhanced value $p=14 \times 10^{17} \mathrm{~cm}^{-3} \mathrm{de}-$ rived from $\mathrm{SdH}$ oscillations. This imbalance could occur if many electrons are pinned in surface states and do not contribute to the conductivity. The observation $p \gg n$ agrees with the recent work of Hoffman et al. ${ }^{14}$ on epitaxial Bi films grown on CdTe. By contrast, the earlier papers by Komnik and co-workers ${ }^{4,5}$ describing $\mathrm{Bi}$ grown on mica claim $n=p$. However, Komnik and co-workers generally used films a few hundreds of nm thick and do mention an excess of holes at low temperatures in their thinnest films. Our holeconcentration values are comparable to those of earlier observations. Following Komnik and co-workers, we take $p=p_{0}+p_{2} / d$, with $p_{0}=2.75 \times 10^{17} \mathrm{~cm}^{-3}$, and find a sur- face state sheet density $p_{2} \approx 6 \times 10^{12} \mathrm{~cm}^{-2}$ from the magnetoconductivity measurements of the $30-\mathrm{nm}$ film and the $\mathrm{SdH}$ results on the thicker films. By comparison, Hoffman et al. and Komnik and co-workers report surface densities of $8 \times 10^{12} \mathrm{~cm}^{-2}$ and $2.5 \times 10^{12} \mathrm{~cm}^{-2}$, respectively. Since the substrate or capping layers should play an important role in the existence and mobility of surface states, it is surprising that similar carrier densities and a predominance of holes are seen in all these experiments.

Most of the fitting parameters are unaffected by the application of pressure. The constancy of the electron and hole mobilities suggests that many more defects are present originally in the 30-nm film than are introduced during sample compression. ${ }^{34}$ As mentioned earlier, a relatively large defect density for the thin films is consistent as well with their negative TCR. The electron concentration also is unchanged by hydrostatic pressure up to $P=20 \mathrm{kbar}$, although a change comparable to the reduction under pressure seen in $\mathrm{SdH}$ measurements on the 500-nm film would fall within the error margins of Table I. Hoffman et al. ${ }^{14}$ found a vanishing of the low-temperature, minority electron concentration in thin epitaxial Bi films as the thickness is reduced below $40 \mathrm{~nm}$. By contrast, our low-temperature 30-nm data indicate a finite electron density, and furthermore, we do not observe any drastic reduction in electron concentration with hydrostatic pressure up to $20 \mathrm{kbar}$.

The hole concentration, on the other hand, is strongly affected by the applied pressure. This is easily seen from the Hall conductivity at various pressures, shown in Fig. 5 for magnetic fields up to $1 \mathrm{~T}$. From Eqs. (1), the slope $d \sigma_{x y} / d B$ is proportional in the low-field limit to the difference $p \mu_{p}-n \mu_{n}$. Since the electron density and the mobilities for both carrier types show little pressure dependence, the slopes of the data in Fig. 5 directly reflect the drop in hole concentration with pressure. At $20 \mathrm{kbar}, p$ has dropped by a factor of 5 to $(3.8 \pm 1.2) \times 10^{17} / \mathrm{cm}^{3}$, a value close to the bulk concentration at zero pressure. This demonstrates that hydrostatic pressure acts as an effective means to remove the surface-induced excess hole concentration.

The precise mechanism for the carrier decrease is unknown. It is likely that pressure affects the band bending at film interfaces. An alternative explanation is that pressure introduces new imperfections which trap carries in local wells. We note, however, that such imperfections, if indeed introduced, would appear to affect the surface-induced carriers without much altering the rest of the film properties. This is supported by our ability to observe $\mathrm{SdH}$ oscillations even 


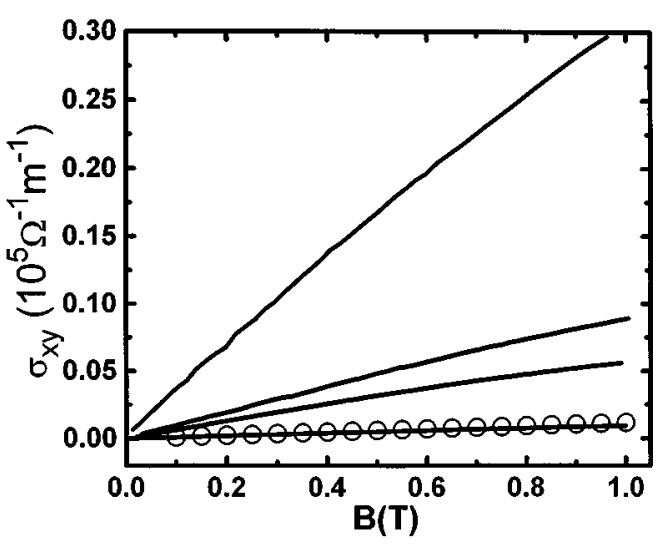

FIG. 5. Low-field Hall conductivity, $\sigma_{x y}$, for the 30-nm-thick film. The traces were taken at $T=1.5 \mathrm{~K}$ and correspond to (top to bottom) zero applied pressure, 2, 4.5, 9, and $20 \mathrm{kbar}$. On the scale of this figure the 20-kbar data are barely distinguishable from the 9-kbar data and are plotted as open circles.

under pressure for our thickest film, and the lack of change in carrier mobility under pressure for the $30-\mathrm{nm}$ film.

The decrease in hole concentration under pressure allows us to comment on the results of Hoffman et al. ${ }^{14}$ Their main argument for a SMSC transition is the temperature dependence of the intrinsic carrier concentration $(n p)^{1 / 2}$. They mention as supporting evidence a suppression of $n$ to less than $(1-2) \times 10^{17} \mathrm{~cm}^{-3}$ at temperatures below $100 \mathrm{~K}$ and thicknesses less than $40 \mathrm{~nm}$. However, when one carrier type is far more common than another, magnetoconductivity fits are insensitive to the exact value of the minority carrier concentration. This applies to our zero-pressure data, where $p \gg n$, as well as to the thin films of the work of Hoffman et al. As we add pressure and reduce $p$, our sensitivity to $n$ increases dramatically. At our higher pressures we unambiguously find $n \approx 2 \times 10^{17} \mathrm{~cm}^{-3}$.

We believe the low-temperature electron density $n$ is finite at zero pressure as well. No carrier concentration has previously been seen to increase with pressure in either bulk or thin-film Bi. In addition to the sharp reduction of $p$ in our 30-nm film, we find a slight reduction of $n$ with pressure in our 500-nm film. Measurements on bulk Bi show a decrease in band overlap under pressure with a corresponding decrease in both $n$ and $p .^{26,27}$ Thus an increase in $n$ with pressure in the 30-nm film would be exactly opposite to the behavior observed in bulk Bi and in our thicker film. We also note that there is no significant change in $n$ with pressure at our higher pressures. By contrast to Ref. 14, our data thus suggest that the $30-\mathrm{nm}$ Bi film has no band gap at zero pressure.

\section{CONCLUSIONS}

We have performed a systematic investigation of the lowtemperature electrical transport properties of singlecrystalline Bi thin films. While the carrier mobilities are still lower than in bulk single crystals, we find that films grown by $\mathrm{MBE}$ on $\mathrm{BaF}_{2}$ substrates show pronounced Shubnikov-de Haas oscillations and, at $500 \mathrm{~nm}$ thickness, retain a bulklike, positive $d R / d T$ at all temperatures. Both aspects attest to the high film quality achievable with the growth process used.

Our results verify earlier reports ${ }^{4,5,14}$ of a term proportional to $1 / d$ in the carrier concentration of $\mathrm{Bi}$ thin films, presumably resulting from a surface contribution. From magnetoconductivity measurements we obtain direct evidence that this extra contribution to the carrier concentration is almost exclusively due to holes. This clearly violates the assumption $n=p$ that has often been retained for the description of the zero-pressure, thin-film limit, as previously pointed out by Hoffman et al. ${ }^{14}$

Excess carriers in thin films have for years prevented direct identification of a thickness-dependent SMSC transition by masking the change in transport properties expected when a band gap appears. Elimination of the minority electrons would be a sign of a band gap, but the dominance of surfaceinduced holes in Bi thin films at zero pressure does not allow accurate extraction of information about the electrons. We circumvent this difficulty by using hydrostatic pressure, which sharply reduces the excess hole concentration. In particular, for a $30-\mathrm{nm}$ film we find that the hole density approaches bulk values at a pressure as low as $9 \mathrm{kbar}$.

The application of pressure, therefore, makes it possible to investigate the SMSC transition directly through magnetotransport measurements. Our results provide evidence that at $30 \mathrm{~nm}$ film thickness and up to $20 \mathrm{kbar}$ the lowtemperature electron density remains finite, indicating that the sample has not undergone any SMSC transition. Measurements on thicker films and bulk samples show that $n$ decreases with pressure. Assuming a similar pressure dependence in the thin film places an upper limit on the critical film thickness at which the SMSC transition occurs in Bi. The most recent estimate, by Hoffman et al. ${ }^{14}$ puts this thickness at $28-30 \mathrm{~nm}$. In view of our data, the SMSC transition, if it indeed exists, would have to occur significantly below $30 \mathrm{~nm}$.

Our results indicate several directions for further experiments. Transport studies under pressure may identify a band gap induced by quantum size effects if thinner, high-quality films can be fabricated. In addition, since size effects are thought to reduce the band overlap, a semimetallic thin film may eventually become semiconducting under pressure. This has been predicted but never directly observed in bulk Bi. We thus believe that pressure studies on MBE-grown, thin bismuth films provide a promising approach towards the investigation of quantum size effects.

\section{ACKNOWLEDGMENTS}

We would like to thank Ronald Griessen and Rinke Wijngaarden for helpful discussions. The work at The University of Chicago was supported in part by the MRSEC Program of the National Science Foundation under Award No. DMR9400379. H.M.J. acknowledges support from the Alfred P. Sloan Foundation and the David and Lucile Packard Foundation. This work was also part of the research program of the "Stichting Fundamenteel Onderzoek der Materie (FOM)," which is financially supported by the "Nederlandse Organisatie voor Wetenschappelijk Onderzoek (NWO)." 
${ }^{1}$ V. B. Sandormirskii, Zh. Éksp. Teor. Fiz. 52, 158 (1967) [Sov. Phys. JETP 25, 101 (1967)]. For a more recent overview, see Y. Imry, in Directions in Condensed Matter Physics, edited by G. Grinstein and G. Mazenko (World Scientific, Singapore, 1986), pp. 101-163.

${ }^{2}$ Yu. F. Ogrin et al., Pis'ma Zh. Éksp. Teor. Fiz. 3, 114 (1966) [JETP Lett. 3, 71 (1966)].

${ }^{3}$ B. A. Tavger and V. Ya. Demikhovskii, Usp. Fiz. Nauk. 96, 61 (1968) [Sov. Phys. Usp. 11, 644 (1969)].

${ }^{4}$ Yu. F. Komnik, E. I. Bukhshtab, Yu. V. Nikitin, and V. V. Andrievskii, Zh. Éksp. Teor. Fiz. 60, 669 (1971) [Sov. Phys. JETP 33, 364 (1971)].

${ }^{5}$ Yu. F. Komnik and V. V. Andrievskii, Fiz. Nizk. Temp. 1, 104 (1975) [Sov. J. Low Temp. Phys. 1, 51 (1975)].

${ }^{6}$ N. O. Birge, B. Golding, and W. H. Haemmerle, Phys. Rev. Lett. 62, 195 (1989); Y. Liu et al., ibid. 67, 2068 (1991).

${ }^{7}$ V. N. Lutskii, Pis'ma Zh. Éksp. Teor. Fiz. 2, 391 (1965) [JETP Lett. 2, 245 (1965)]; V. N. Lutskii and L. A. Kulik, ibid. 8, 133 (1968) [ibid. 8, 80 (1968)].

${ }^{8}$ H. T. Chu and W. Zhang, J. Phys. Chem. Solids 53, 1059 (1992).

${ }^{9}$ N. Garcia et al., Phys. Rev. B 5, 2029 (1972).

${ }^{10}$ H. T. Chu et al., Phys. Rev. B 37, 3900 (1988); 45, 11233 (1992).

${ }^{11}$ I. Goldfarb and B. Tavger, Fiz. Tverd. Tela (Leningrad) 11, 1517 (1969) [Sov. Phys. Solid State 11, 1231 (1969)]; M. I. Kaganov, S. S. Nedorezov, and A. M. Rustamova, Fiz. Tverd. Tela (Leningrad) 12, 2277 (1970) [Sov. Phys. Solid State 12, 1820 (1971)].

${ }^{12}$ H. T. Chu, J. Phys. Chem. Solids 48, 845 (1987); 50, 319 (1989); 50, 1121 (1989); H. T. Chu and Tian Li, ibid. 53, 45 (1992).

${ }^{13}$ T. Zakrzenski and E. Z. Oziuba, Phys. Status Solidi B 52, 665 (1972).

${ }^{14}$ C. A. Hoffmann et al., Phys. Rev. B 48, 11431 (1993).

${ }^{15}$ R. G. P. van der Kraan et al., Phys. Rev. B 44, 13140 (1991).

${ }^{16}$ D. L. Partin et al., Phys. Rev. B 38, 3818 (1988); D. L. Partin et al., J. Vac. Sci. Technol. B 7, 348 (1989).

${ }^{17}$ J. A. van Hulst, H. M. Jaeger, and S. Radelaar, Phys. Rev. B 52, 5953 (1995).

${ }^{18}$ G. E. Smith, G. A. Baraff, and J. M. Rowell, Phys. Rev. 135, A1118 (1964); G. E. Smith, L. C. Hebel, and S. J. Buchsbaum, ibid. 129, 154 (1963).
${ }^{19}$ R. N. Zitter, Phys. Rev. 127, 1471 (1962).

${ }^{20}$ P. W. Bridgman, Proc. Am. Acad. Arts Sci. 81, 165 (1952).

${ }^{21}$ R. M. Overney et al., Surf. Sci. Lett. 277, L29 (1992).

${ }^{22}$ J. A. van Hulst, Ph.D. thesis, Delft University of Technology, 1995.

${ }^{23}$ S. A. Carter, Ph.D. thesis, The University of Chicago, 1993.

${ }^{24}$ For pressure calibration we use $d T_{c} / d P=-0.039 \mathrm{~K} / \mathrm{kbar}$ from $\mathrm{T}$. F. Smith and C. W. Chu, Phys. Rev. 159, 353 (1967).

${ }^{25}$ L. J. van der Pauw, Philips Res. Rep. 13, 1-9 (1958).

${ }^{26}$ D. Balla and N. B. Brandt, Zh. Éksp. Teor. Fiz. 47, 1653 (1964) [Sov. Phys. JETP 20, 1111 (1965)]; E. S. Itskevich and L. M. Fisher, Zh. Éksp. Teor. Fiz. 53, 98 (1967) [Sov. Phys. JETP 26, 66 (1968)].

${ }^{27}$ N. B. Brandt, V. V. Moschalkov, and S. M. Chudinov, Zh. Éksp. Teor. Fiz. 74, 1829 (1978) [Sov. Phys. JETP 47, 953 (1978)].

${ }^{28}$ L. M. Roth and P. N Agyres, in Semiconductors and Semimetals, edited by R. K. Willardson and A. C. Brer (Academic, New York, 1966), Vol. 1, pp. 184 and 185.

${ }^{29}$ We have observed this behavior even more clearly in the 50-nmthick film using fields up to $13 \mathrm{~T}$ (Ref. 22).

${ }^{30}$ R. Hartman, Phys. Rev. 181, 1070 (1969).

${ }^{31}$ M. J. Kane et al., J. Phys. C 18, 5629 (1985).

${ }^{32}$ M. A. Reed, W. P. Kirk, and P. S. Kobiela, IEEE J. Quantum Electron. QE-22, 1753 (1986).

${ }^{33}$ E. F. Schubert, K. Ploog, H. Dämbkes, and K. Heime, Appl. Phys. A 33, 63 (1984).

${ }^{34}$ The values we obtain for mobilities are lower than those found in epitaxial $\mathrm{Bi} / \mathrm{Bi}_{1-x} \mathrm{Sb}_{x}$ heterostructures grown on $\mathrm{BaF}_{2}$ with $\mathrm{Bi}$ layers of the same thickness (Ref. 17; the conduction in the semiconducting $\mathrm{Bi}_{1-x} \mathrm{Sb}_{x}$ buffer layers freezes out at low temperatures). This is probably due to a less perfect lattice match with the substrate and the Si capping layer, and a corresponding increase in defects. $\mathrm{Bi} / \mathrm{Bi}_{1-x} \mathrm{Sb}_{x}$ heterostructures, on the other hand, are not well suited for an investigation of the effects of pressure on the $\mathrm{Bi}$ layer since the carrier reduction in $\mathrm{Bi}$ is accompanied by an unknown change in carrier density in $\mathrm{Bi}_{1-x} \mathrm{Sb}_{x}$ under applied pressure. 